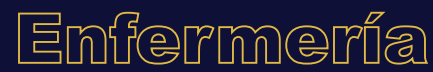 \\ Unกtversitara
}

\section{Conductas sexuales de riesgo para embarazos no deseados e infecciones de transmisión sexual en estudiantes universitarios}

\section{Risky sexual behaviors related to unwanted pregnancies and sexually transmitted infections among university students}

\section{Condutas sexuais de risco para gravidez não desejada e infecções de transmissão sexual em estudantes universitários}

\author{
H. Tapia-Martínez ${ }^{\mathrm{a}^{*}}$, J. Hernández-Falcón ${ }^{\mathrm{b}}$, I. \\ Pérez-Cabrerac ${ }^{c}$ A. Jiménez-Mendoza ${ }^{\mathrm{d}}$ \\ ORCID \\ ${ }^{\mathrm{a}} 0000-0003-4998-3506$ \\ b $0000-0002-0614-4342$ \\ ${ }^{c} 0000-0002-5068-6325$ \\ d0000-0001-5762-4314
}

Universidad Nacional Autónoma de México, Escuela Nacional de Enfermería y Obstetricia, División de Estudios Profesionales, Ciudad de México, México

Recibido: 27 junio 2019

Aceptado: 10 agosto 2020

RESUMEN

Introducción: Los adolescentes como grupo vulnerable al tratar de satisfacer su sexualidad se exponen a conductas sexuales de riesgo (CSR) que contribuyen al incremento de Embarazos No Deseados (END) e Infecciones de Transmisión Sexual (ITS).

Objetivo: Describir las conductas de riesgo sexual en jóvenes universitarios de la Escuela Nacional de Enfermería y Obstetricia, Universidad Nacional Autónoma de México

\footnotetext{
*Autor para correspondencia. Correo electrónico: htaapiag9@yahoo.com https://doi.org/10.22201/eneo.23958421e.2020.3.703 
Métodos: Estudio cuantitativo, descriptivo, transversal y correlacional; población, 580 estudiantes de primer semestre (febrero-junio 2017); muestra, 144 sujetos elegidos al azar; análisis con estadística descriptiva e inferencial; la participación de los estudiantes fue anónima, voluntaria y los datos totalmente confidenciales.

Resultados: $78 \%$ fueron mujeres y $72 \%$ con edades de $18-19$ años; presencia de CSR: cuatro de cada cinco han tenido vida sexual; 80\% 2-3 o más veces; 81\% sexo sin protección, $48 \%$ 2 o más veces; $60 \%$ uso de anticonceptivos naturales, $35 \%$ dos o más ocasiones; $50 \%$ sexo bajo la ingestión de alcohol o drogas, $43 \%$ una, dos o más veces y; $39 \%$ errores en el uso de métodos anticonceptivos, $11 \%$ dos o más ocasiones. Existe una correlación significativa entre la actividad sexual y la experiencia con anticonceptivos, así como de actividad sexual con la conducta sexual en condiciones de riesgo.

Discusión y Conclusiones: Los resultados coinciden con estudios previos. Resulta importante identificar las CSR en los estudiantes universitarios para priorizar estrategias de salud sexual. Los profesionales de enfermería responsables de la Consejería Sexual tienen un rol fundamental en la educación sexual.

Palabras clave: Conducta sexual; estudiantes universitarios; embarazo en adolescencia; infecciones de transmisión sexual; México.

\section{ABSTRACT}

Introduction: Adolescents can engage into risky sexual behaviors which can increase unwanted pregnancies and sexually transmitted infections (STI).

Objective: To describe the risky sexual behaviors among university students belonging to the Escuela Nacional de Enfermería y Obstetricia, Universidad Nacional Autónoma de México.

Methods: This is a quantitative, descriptive, transversal and correlational study with a population of 5801 1st semester students (February-June 2017). The sample was constituted by 144 randomly selected students. Descriptive and inferential statistics were calculated. Participation was anonymous and all data were kept strictly confidential.

Results: 78\% were female. 72\% were 18-19 years old. Regarding their sexual life, four of five reported having had some form of sexual activity, with $80 \%$ saying 2 or more times. $81 \%$ had unprotected sex, with $48 \%$ saying two or more times. $60 \%$ reported the use of natural contraceptives, with $35 \%$ saying two or more times. $50 \%$ said having had sex under the influence of drugs or alcohol, with $43 \%$ saying two or more times. $39 \%$ acknowledged errors while using contraceptive methods, with $11 \%$ A significant correlation was found between sexual activity and experience in the use of contraceptives, as well as between sexual activity and risky sexual behaviors.

Discussion and Conclusions: These findings turned out to be similar to those of previous studies. It is important to identify sexual risky behaviors among university students in order to prioritize sexual health strategies. Nursing professionals who are responsible for Sex-Related Advice have a key role in sex education.

Keywords: Sexual behavior; university students; pregnancy in adolescence; sexually transmitted infections; Mexico. 


\section{RESUMO}

Introdução: Os adolescentes como grupo vulnerável na tentativa de satisfazer sua sexualidade estão expostos a condutas sexuais de risco (CSR) que contribuem para o aumento da Gravidez Não Desejada (END) e Infecções de Transmissão Sexual (ITS).

Objetivo: Descrever as condutas de risco sexual em jovens universitários da Escuela Nacional de Enfermería y Obstetrícia, Universidad Nacional Autonoma de México.

Métodos: Estudo quantitativo, descritivo, transversal e correlacional; população, 580 estudantes de primeiro semestre (fevereiro-junho 2017); amostragem, 144 sujeitos escolhidos aleatoriamente; análise com estatística descritiva e inferencial; a participação dos estudantes foi anónima, voluntária e os dados totalmente confidenciais.

Resultados: 78\% foram mulheres e 72\% com idades de 18-19 anos; presência de CSR: quatro em cada cinco já tiveram vida sexual; 80\% 2-3 ou mais vezes; 81\% sexo desprotegido, $48 \%$ 2 ou mais vezes; $60 \%$ uso de anticonceptivos naturais, $35 \%$ duas ou mais ocasiões; $50 \%$ sexo sob ingestão de álcool ou drogas, $43 \%$ uma, duas ou mais vezes e; $39 \%$ erros no uso de métodos anticoncepcionais, $11 \%$ duas ou mais ocasiões. Existe uma correlação significativa entre a atividade sexual e a experiência com contraceptivos, bem como a atividade sexual com a conduta sexual em condições de risco.

Discussão e Conclusões: Os resultados coincidem com estudos prévios. Resulta importante identificar as CSR nos estudantes universitários para priorizar estrategias de saúde sexual. Os profissionais de enfermagem responsáveis pelo Aconselhamento Sexual têm um papel fundamental na educação sexual.

Palavras chave: Comportamento sexual; estudantes universitarios; gravidez na adolescência; infecções sexualmente transmissiveis; México.

\section{INTRODUCCIÓN}

Los estudiantes universitarios de primer año de la Escuela Nacional de Enfermería y Obstetricia (ENEO), como grupo vulnerable integrado por adolescentes y jóvenes en edades comprendidas de 18 a 24 años se hallan expuestos a factores que los llevan a practicar conductas sexuales de riesgo que favorecen el incremento de Embarazos No Deseados (END) e Infecciones de Transmisión Sexual (ITS), al intentar satisfacer su sexualidad.

La Conducta Sexual de Riesgo (CSR) se define como la exposición del individuo a una situación que puede ocasionar daños a su salud o a la de otra persona ${ }^{1}$. Dicha exposición se ubica en tres aspectos: en primer lugar, la falta de uso de métodos anticonceptivos de barrera (en específico, el condón); en segundo lugar, múltiples parejas; por último, la inclusión de actividades que facilitan el intercambio de fluidos entre la pareja, o actividades con las cuales no resulta fácil tomar buenas decisiones, por ejemplo, tener actividad sexual bajo intoxicación por alcohol o estupefacientes ${ }^{2}$ o, el rechazo a buscar atención médica para las infecciones de transmisión sexual33.

Las consecuencias de las CSR en estudiantes universitarios pueden interrumpir las metas educativas o poner en riesgo la vida de los individuos. En efecto, un embarazo como producto de la exposición a CSR tiene un efecto profundo en la trayectoria de vida de estas personas, obstaculiza su desarrollo psicosocial, se asocia con resultados deficientes en materia de salud tanto para la madre 
como para sus hijos, repercute negativamente en sus oportunidades educativas y laborales, por lo que contribuye a perpetuar los ciclos intergeneracionales de pobreza y mala salud4.

Si bien existen programas a nivel internacional y nacional para evitar o prevenir embarazos a temprana edad e ITS como: las Directrices de la Organización Mundial de la Salud (OMS) para la prevención del embarazo precoz en 20125; el Programa Prevención del embarazo precoz del UNFPA del $2014^{6}$ y; en México la Estrategia Nacional para la Prevención del Embarazo en Adolescentes (ENAPEA) de la Secretaria de Salud de México $2015^{7}$, el embarazo en la adolescencia persiste e inclusive sigue en aumento.

Según la OMS, 16 millones de mujeres de 15 a 19 años y aproximadamente un millón de niñas menores de 15 años dan a luz cada año, la mayoría en países de ingresos bajos y medianos ${ }^{8}$. Las tasas de embarazo adolescente en América Latina están entre las más altas del mundo, después de África subsahariana y el sur de Asia, de acuerdo con un estudio del Banco Mundial, en 2010 se registraron en la región latinoamericana 72 nacimientos por cada 1000 en mujeres de 15 a 19 años, a diferencia de África que presentó 1089. En México la tasa de fecundidad para este grupo ha pasado de 69.2 nacimientos por cada 1000 mujeres en 1997, a 70.9 en el 2009 y 77 en $2014^{10}$. Esta cifra supera la de América Latina.

Las ITS son otra consecuencia como producto de la CSR. La OMS refiere que cada día más de 1 millón de personas contraen esta clase de infección, por lo que se estima que, anualmente, unos 376 millones de personas contraen alguna de las cuatro ITS siguientes: clamidiasis, gonorrea, sífilis y tricomoniasis; más de 500 millones de personas son portadoras del virus que provoca el herpes genital tipo 2 (HSV2) y aproximadamente 290 millones de mujeres están infectadas con el virus del papiloma humano $(\mathrm{VPH})^{11}$.

De acuerdo con el Consejo Nacional de Población (CONAPO), en el 2015 México contaba con una población de 122 millones de personas, de las cuales 59 millones eran hombres y 62 millones eran mujeres ${ }^{12}$. En ese entonces hubo 715089 casos de ITS; las mujeres presentaron el porcentaje mayor con 96.5\%, es decir, 689987 casos. El grupo de edad más afectado fue el de 15 a 44 años con 560676 casos; etapa de vida donde las personas se encuentran con mayor posibilidad de ser productivas para la sociedad y de tener una vida sexual y reproductiva sana. De este conjunto se destaca el grupo de 15 a 24 años que incluye la adolescencia y la juventud, en el cual hubo 200488 casos $(28 \%)^{13}$.

Los Objetivos del Desarrollo Sostenible preparados por la ONU para los próximos 15 años sobre salud y bienestar (objetivo 3) y el referente a igualdad de género (objetivo 5) ${ }^{14}$, están encaminados a la solución de esta problemática, pretenden anticiparse a problemas de embarazos precoces como consecuencia de las necesidades actuales no satisfechas en anticoncepción, tasas elevadas de VIH y sida, una educación sexual escasa o inexistente o el matrimonio infantil15.

La población de 10 a 19 años es la más afectada por END e ITS, los jóvenes universitarios de 18 a 19 años, como parte de ese grupo, no escapan a dicha problemática. En la Universidad Nacional Autónoma de México (UNAM) en 2015, se aplicó el Examen Médico Automatizado (EMA) en población de nuevo ingreso que incluyó 32,859 alumnos, de los cuales 3.8\% había experimentado un END (1,248 estudiantes) y el 1\% había tenido una ITS (328); asimismo el 31.2\% $(10,252)$ refirieron tener vida sexual sin utilizar un método anticonceptivo y un 40.9\% de los estudiantes utilizó la píldora de emergencia $(13,439)^{16}$.

Con relación a la ENEO, los resultados del EMA aplicado en el 2014 a 507 alumnos arrojaron que el 11.7\% (59) de los estudiantes experimentaron un END antes de entrar a la carrera; el 2.1\% (10) mencionó haber padecido una ITS; 40.4\% de los jóvenes tenían actividad sexual sin el uso de algún 
método anticonceptivo o que los proteja de ITS (204), esto representa 10\% más que la población general de la UNAM; el 38.4\% de la población de nuevo ingreso recurrió a un método de emergencia (194); asimismo, el $46.1 \%$ de los jóvenes tenía un alto y constante consumo de alcohol (233) ${ }^{17}$, lo cual aumenta el riesgo de END e ITS.

Las CSR en jóvenes han sido estudiadas en población universitaria pública y privada, se señalan entre las más frecuentes y significativas: el consumo de bebidas alcohólicas, la ingesta de drogas ilícitas, la actividad de sexo no protegido, la falta de planificación en las relaciones sexuales, el número de parejas y la baja disponibilidad de preservativos para llevar a cabo prácticas seguras; las cuales pueden estar determinadas por factores internos y externos que pueden favorecer una disminución del control de la situación y mayor desprotección al virus de la inmunodeficiencia humana (VIH), otras ITS y END 2,18 .

Ante este panorama surgió la pregunta de investigación: ¿Cuáles son las conductas sexuales de riesgo para embarazos no deseados e infecciones de transmisión sexual en estudiantes universitarios? Y como objetivo de investigación, describir las conductas sexuales de riesgo para END e ITS en estudiantes universitarios.

\section{MÉTODOS}

Diseño de estudio: el estudio fue de tipo cuantitativo, transversal, descriptivo y correlacional. La población la conformaron 580 estudiantes de la ENEO de la UNAM de primer semestre de febrero a junio de 2017, en ambos turnos, de las dos licenciaturas; la muestra fue probabilística aleatoria con un tamaño de 144 sujetos de estudio, la cual se calculó con la fórmula para poblaciones finitas ${ }^{19}$.

Criterios de inclusión: estudiantes del primer año de las Licenciaturas de Enfermería, Enfermería y Obstetricia; con edades entre 18 y 24 años que aceptaran participar.

Criterios de exclusión: estudiantes que cursaban el segundo, tercero o cuarto año; mayores de 24 años y quienes recursaron el primer año.

Criterios de eliminación: estudiantes que no completaron el instrumento.

Para la recolección de datos se aplicó el instrumento Censo SSEU-YO (sobre el sexo entre los estudiantes universitarios) de Pulido et al., un cuestionario autoadministrado que evalúa la CSR de la persona que contesta; tiene una confiabilidad de .815 Alpha de Cronbach y una validez de .854 por el análisis de correlación de Spearman-Brown ${ }^{20}$. Consta de 27 reactivos, para efectos de este estudio se agregaron 4 preguntas demográficas, haciendo un total de 31 ítems. Es un censo en pares de preguntas cerradas con cuatro opciones de respuesta (últimos 30 días, últimos 12 meses, alguna vez, nunca), las preguntas pares evalúan la ausencia o presencia de la conducta sexual de riesgo (reactivos $8,10,12,14,16,18,20,22,24$ y 26), y las preguntas impares miden la frecuencia en número de veces; por ejemplo: tres o más veces, dos veces, una vez y nunca de esa conducta (reactivos 7, 9, $11,13,15,17,19,21,23,25$ y 27). Asimismo, el instrumento evalúa 5 dimensiones: primera, actividad sexual (reactivo 6); segunda, utilización de métodos anticonceptivos, sexo sin protección, errores en su uso y métodos naturales (reactivos: 8, 20 y 22); tercera, sexo bajo la influencia de alcohol o drogas donde el sujeto ha tenido sexo después de haber consumido alcohol o drogas (reactivo 18); cuarta, sexo casual cuando el sujeto ha tenido sexo con una persona a la que acaba de conocer (reactivos, 14, 16, 26) y; quinta, historia sexual que recopila información sobre las actividades sexuales que ha realizado el sujeto hasta la fecha de aplicación del cuestionario (reactivos: 10, 12 y 24).

Los cuestionarios se aplicaron en forma grupal en los salones seleccionados aleatoriamente, cuyo grupo fue posible localizar y en los que el profesor y los estudiantes accedieron a participar, 
previa explicación de la finalidad de la investigación. Se aseguró la confidencialidad en el manejo de los datos.

El estudio se basó en el marco establecido en el Reglamento de la Ley General de Salud en los artículos 34, 36, 38 y 39 que se refieren al informe de riesgo mínimo para los participantes del estudio, el consentimiento informado, el anonimato y la libertad de retirarse en cualquier momento del estudio, así como los principios éticos de beneficencia y no maleficencia, la veracidad, la privacidad, confidencialidad, autonomía, equidad y justicia que se indica en el Código de Ética de la enfermería, principios éticos de la ENEO y Ley General de los Derechos de Niñas, Niños y Adolescentes incluidos en la Comisión Nacional de Derechos Humanos ${ }^{21-24}$. La investigación fue aprobada por el Comité de Ética e Investigación de la ENEO-UNAM con el número de registro 101 Proyecto Efecto de una intervención de consejería en las conductas sexuales de riesgo en estudiantes universitarios.

Para el procesamiento de datos se utilizó el programa SPSS v.2O. En el análisis se usaron estadísticas descriptivas para resumir los datos de las 5 principales variables, tanto de ausenciapresencia y frecuencia de la CSR, el resto resultó irrelevante; y estadística inferencial para medir la correlación de la variable actividad sexual (aquellos con vida sexual), con las variables experiencia en anticonceptivos y sexo bajo condiciones de riesgo.

\section{RESULTADOS}

\section{a) Datos demográficos}

De los 144 alumnos participantes el 78\% correspondió a mujeres y 22\% a hombres, el $72 \%$ de la muestra perteneció al rango de edad de 18-19 años y el 19\% al de 20 a 21, con una media de 20.5 años. El 79\% correspondió a la Licenciatura en Enfermería y Obstetricia y el 21\% a la Licenciatura en Enfermería; sobre el bachillerato de procedencia, el 41\% correspondió al $\mathrm{CCH}, 35 \%$ a la preparatoria UNAM y el 10\% al Colegio de Bachilleres.

\section{b) Variable ausencia/presencia de la CSR}

De acuerdo con el instrumento SSEU-YO se obtuvieron los siguientes resultados: casi la mitad de los universitarios encuestados (48\%) reportaron haber tenido relaciones sexuales en los últimos 30 días, un tercio, el $24 \%$ en los últimos 12 meses y 9\% alguna vez; en otros términos, cuatro de cada cinco han iniciado y/o han tenido vida sexual.

El 81\% de los estudiantes que han tenido relaciones sexuales (RS) las han tenido sin protección, ya sea alguna vez, en el último año o en los últimos 30 días. El uso de métodos anticonceptivos naturales como el coito interrumpido y método del calendario son las estrategias reportadas por esta población con objeto de evitar el embarazo, 15\% lo usaron en los últimos 30 días, $13 \%$ en los últimos 12 meses y $32 \%$ alguna vez.

En cuanto al uso de alcohol u otras sustancias ligado al hecho de tener relaciones sexuales, un 40\% manifestó que esto ocurrió alguna vez, si a esto se suma quienes lo hicieron así en los últimos 12 meses o en los últimos 30 días, la mitad de estos estudiantes que han tenido RS lo han realizado en estas condiciones. Por otra parte, $39 \%$ en total han tenido en algún momento errores con el uso de anticoncepción en sus RS (Tabla 1).

Otras conductas de riesgo evaluadas se presentaron en porcentajes muy bajos como el sexo casual, o con personas diferentes a la pareja, o con prostitutas. 
Tabla 1. Presencia de Conductas sexuales de riesgo (porcentajes, $n=117^{*}$ )

\begin{tabular}{lcccc}
$\begin{array}{l}\text { Conductas sexuales } \\
\text { de riesgo }\end{array}$ & 30 días & 12 meses & Alguna vez & Nunca \\
\hline $\begin{array}{l}\text { Relaciones Sexuales } \\
\text { sin protección }\end{array}$ & 34.5 & 18.5 & 27.1 & 19.7 \\
$\begin{array}{l}\text { Uso anticonceptivos } \\
\text { naturales }\end{array}$ & 14.8 & 13.5 & 32.0 & 39.3 \\
$\begin{array}{l}\text { RS con uso alcohol/ } \\
\text { otras sustancias }\end{array}$ & 4.9 & 6.2 & 40.6 & 48.7 \\
$\begin{array}{l}\text { Errores en uso de } \\
\text { anticoncepción }\end{array}$ & 2.6 & 5.1 & 31.0 & 61.5 \\
\hline
\end{tabular}

* Se excluyeron a quienes nunca han tenido relaciones sexuales

a Se consideraron como tales el coito interrumpido y el método del calendario

\section{c) Variable frecuencia en veces de la CSR}

Considerando sólo al grupo de estudiantes con antecedentes de relaciones sexuales, alrededor del $50 \%$ las han tenido tres o más veces, lo que pudiera corresponder a ser activos sexualmente; quienes han practicado el sexo sin protección 2 o más veces corresponden al 48\%; por su parte, los que refieren el hecho uso de anticonceptivos naturales como estrategia para evitar embarazos en 2 o más veces, son más de una tercera parte. En lo que respecta al sexo bajo la ingestión de alcohol o drogas, una cuarta parte lo hizo sólo una vez, este porcentaje disminuye a la mitad cuando lo han hecho dos veces y, únicamente el $4.3 \%$ lo han realizado tres veces o más. Sobre los errores en el uso de métodos anticonceptivos, $11 \%$ los tuvieron 2 у 3 o más veces (Tabla 2).

\begin{tabular}{|c|c|c|c|c|}
\hline $\begin{array}{c}\text { Conductas sexuales } \\
\text { de riesgo }\end{array}$ & 3 ó mas veces & 2 veces & Una vez & Nunca \\
\hline $\begin{array}{l}\text { Relaciones Sexuales } \\
\text { sin protección }\end{array}$ & 29.1 & 18.8 & 17.1 & 35.0 \\
\hline $\begin{array}{l}\text { Uso anticonceptivos } \\
\text { naturales }\end{array}$ & 26.5 & 8.6 & 12.8 & 52.1 \\
\hline $\begin{array}{l}\text { RS con uso alcohol/ } \\
\text { otras sustancias }\end{array}$ & 4.3 & 12.8 & 25.6 & 57.3 \\
\hline $\begin{array}{l}\text { Errores en uso de } \\
\text { anticoncepción }\end{array}$ & 3.4 & 7.7 & 20.5 & 68.4 \\
\hline
\end{tabular}

* Se excluyeron a quienes nunca han tenido relaciones sexuales

a Se consideraron como tales el coito interrumpido y el método del calendario

\section{d) Correlaciones entre actividad sexual con experiencia en anticonceptivos y actividad sexual con sexo bajo condiciones de riesgo}

De acuerdo con el coeficiente de correlación de Spearman se encontró que existe una correlación significativa entre: la actividad sexual sin protección anticonceptiva o el uso de métodos menos 
confiables (sexo sin protección, coito interrumpido o método del calendario) de 0.7970 y actividad sexual con sexo bajo condiciones de riesgo (sexo bajo la influencia de alcohol o drogas) de 0.4516 .

La actividad sexual se correlaciona con el sexo sin protección, coito interrumpido o método del calendario; sin embargo, el estudio no alcanza a discriminar las características de los anticonceptivos usados (métodos de barrera, anticonceptivos hormonales, etc.), ni si el uso de los mismos es el más adecuado. La segunda correlación es aún más preocupante ya que muestra que la actividad sexual se está practicando bajo la influencia de alcohol o drogas.

\section{DISCUSIÓN}

Este estudió permitió la descripción de las CSR en población estudiantil universitaria de primer año, en el cual se brinda una imagen que integra variables sociodemográficas que incluyen el sexo, la edad, la licenciatura que estudian y el bachillerato de procedencia, así como las variables que corresponden a las CSR, bajo la perspectiva de instrumento utilizado (Censo SSEU-YO), que midió la presencia/ausencia y la frecuencia en veces de la conducta sexual de riesgo. Dado que en la ENEO se preparan las futuras generaciones del país, el grupo de estudio fueron estudiantes (hombres y mujeres) de recién ingreso a los estudios universitarios, con el fin de identificar las necesidades más apremiantes para favorecer el ejercicio de la sexualidad con los menores riesgos.

En este grupo, como es habitual en esta carrera, hubo predominio de mujeres, en edades que abarcan la etapa de adolescencia-juventud conforme a los criterios de inclusión y aun cuando ya están en nivel universitario tienen altos porcentajes en CSR como el sexo sin protección e incluso; una fuerte correlación entre la actividad sexual con baja experiencia en uso de anticonceptivos o uso de métodos de baja seguridad (sexo sin protección, coito interrumpido o método del calendario). Esto último puede desencadenar problemas como el END o ITS y en consecuencia, deserción y/o bajo rendimiento escolar, que favorece, como ha sido señalado en la literatura, la repetición del ciclo de pobreza y mala salud.

De esta manera, los resultados más relevantes de este estudio indicaron que el $72 \%$ de los sujetos son mujeres de 18 y 19 años en una carrera predominantemente femenina, asumiendo que la condición de ser mujer, aunque estén estudiando en la universidad, aún está influida por estereotipos que las hacen vulnerables a tener CSR, como se observó en otro estudio realizado en estudiantes de octavo semestre de Enfermería y Obstetricia pertenecientes a la ENEO. En cuyo caso se señaló que persisten algunos estereotipos de género, como una actitud sumisa de las mujeres, no querer preocupar a su pareja si cree estar embarazada (20\%), o pensar que cuando su pareja no tiene deseo sexual le interesa otra mujer (25\%); igualmente más del $24 \%$ de las jóvenes encuestadas reflejó una actitud pasiva (dejarse llevar) en su relación y el 25.8\% ve los celos como normales en los novios 25 .

Las CSR para END e ITS mostraron que 4 de cada 5 de los participantes son sexualmente activos, es decir, el $81 \%$ ha tenido relaciones sexuales por lo menos alguna vez en su vida y más de la mitad las realiza con una frecuencia de 3 a más veces; de igual forma, más de la mitad refirió sexo sin protección. Al respecto, tres estudios internacionales coinciden con estos resultados, los cuales mencionan que la mayoría de los estudiantes eran sexualmente activos y la tasa sobre el uso de anticoncepción seguía siendo baja ${ }^{26-28}$.

Casi la mitad de los estudiantes tuvieron sexo bajo la ingestión de alcohol o drogas e hicieron uso de métodos naturales, mientras que una cuarta parte manifestó haber tenido errores en el uso de métodos anticonceptivos. Cabe destacar que dichos resultados coinciden con dos estudios a nivel 
nacional y dos a nivel internacional en los cuales se proponen como factor predisponente para los END e ITS el consumo de bebidas alcohólicas, ingesta de drogas ilícitas, la actividad de sexo no protegido, falta de planificación en las relaciones sexuales, número de parejas y baja disponibilidad de preservativos para llevar a cabo una salud sexual ${ }^{1,20,28,29}$.

Asimismo, existe una correlación significativa entre la actividad sexual y la experiencia baja o ausente con anticonceptivos, que se expresa en tener sexo sin protección, coito interrumpido o uso de método del calendario; no se encontraron otros estudios que hayan también realizado esta correlación. Sin embargo, la actividad sexual y conducta sexual en condiciones de riesgo o relaciones sexuales bajo la influencia de alcohol o drogas también tuvo una correlación importante y coincide con los resultados de otros estudios a nivel internacional ${ }^{30,31}$.

Por lo tanto, la educación sobre salud sexual puede ayudar a los estudiantes de enfermería a mejorar sus conocimientos, a explorar sus propios valores, y del mismo modo, es importante en su preparación académica para cumplir los desafíos en el futuro al brindar atención en aspectos relacionados con la salud sexual y reproductiva ${ }^{32}$.

Una limitación del estudio es que se realizó en un solo contexto, el universitario. Se proponen estudios comparativos con otros contextos, otras carreras universitarias y otros países. Una muestra más heterogénea respecto a la edad y en estudiantes de distintos niveles semestrales permitiría conocer de forma más global las CSR.

\section{CONCLUSIONES}

Con base en lo anterior, el estudio muestra una población expuesta al riesgo de END o ITS ya que la mayoría es sexualmente activa, no practica medidas de protección y son jóvenes aún vulnerables. En este contexto resulta importante identificar las CSR en los estudiantes universitarios para priorizar estrategias de salud sexual, reforzando la formación sobre salud sexual y reproductiva con el propósito de lograr prácticas seguras y libres de riesgo.

Los profesionales de enfermería tienen un rol fundamental en la educación sexual de los estudiantes, puesto que realizan actividades relacionadas con la promoción y consejería sobre conductas sexuales saludables. En este sentido, los profesionales de enfermería responsables de la Consejería Sexual tienen un papel esencial en la educación sexual reforzando la formación en los estudiantes universitarios sobre salud sexual y reproductiva, favoreciendo las prácticas seguras y libres de riesgo para ser transmisores y consejeros en esta área.

\section{RESPONSABILIDADES ÉTICAS}

Protección de personas y animales. En este estudio no se realizaron experimentos con personas o animales.

Confidencialidad. En esta investigación no aparecen datos personales que permitan identificar a los participantes, a quienes se les garantizó el anonimato y la confidencialidad de la información.

Conflicto de intereses. Los autores declaran no tener conflicto de intereses.

Financiamiento. El financiamiento de este artículo estuvo a cargo de la Fundación Río Arronte de México con el código S.582. 


\section{REFERENCIAS}

1. Gonçalves-Câmara S, Castellá-Sarriera J, Carlotto MS. Predictores de conductas sexuales de riesgo entre adolescentes. Interam J. Psychol. 2007; 41(2): 161-6.

2. Forcada-Mier P, Pacheco-Murguía AS, Pahua-Mendoza E, Pérez-Palacios Rodríguez P, Todd-Flores NE, Pulido-Rull MA. Conducta sexual de riesgo en estudiantes universitarios: factores de riesgo y protección. Rev. intercont. psicol. educ. 2013; 15(1): 23-46.

3. Organización Panamericana de la Salud. Estrategia y plan de salud regional sobre los adolescentes y jóvenes 2010-2018. Washington, D.C.: OPS; 2010. https://bit.ly/1MxgnoI

4. Organización Panamericana de la Salud / Organización Mundial de la Salud / Fondo de Población de las Naciones Unidas / Fondo de las Naciones Unidas para la Infancia. Acelerar el progreso hacia la reducción del embarazo en la adolescencia en América Latina y el Caribe. Informe de consulta técnica. Washington, D.C.: OPS; 2018. https://ng.cl/xcoma

5. Organización Mundial de la Salud. Directrices de la OMS para la prevención del embarazo precoz y de los resultados reproductivos adversos en adolescentes en los países en desarrollo. Ginebra: OMS/UNFPA; 2012. https://bit.ly/2KJ5IoO

6. Fondo de Población de las Naciones Unidas. Embarazo Adolescente. Nueva York: UNFPA; 2014. https://bit.ly/2Iy5a2r

7. Secretaría de Salud. Estrategia Nacional para la prevención del embarazo en adolescentes. México: SSA; 2015. https://bit.ly/2lbKDVJ

8. Organización Mundial de la Salud. El embarazo en la adolescencia. Ginebra: OMS; 2014 https://bit.ly/1EDJsP5

9. Banco Mundial. Cada vez más jóvenes latinoamericanas llegan a los quince años con un embarazo. Washington, D.C.: Banco Mundial; 2013. https://bit.ly/2WScRL7

10. Instituto Nacional de Estadística y Geografía/Consejo Nacional de Población. Encuesta Nacional de la Dinámica Demográfica ENADID 2014. México: INEGI/CONAPO; 2015. https://bit.ly/2W59WZ4

11. Organización Mundial de la Salud. Infecciones de transmisión sexual. Ginebra; OMS: 2019. https://bit.ly/1EDJsP5

12. Consejo Nacional de Población. Proyecciones de la Población de México 2010-2050. México: CONAPO; 2012.

13. Secretaría de Salud. Anuarios de Morbilidad 1984- 2019. México: SSA; 2014.

14. Organización de las Naciones Unidas. Objetivos del desarrollo Sostenible. 17 objetivos para transformar nuestro mundo. Nueva York: ONU; 2016. http://bit.ly/1CrYw5O

15. Fondo de Población de las Naciones Unidas. Estado de la Población Mundial 2016. Nueva York: UNFPA; 2016. http://bit.ly/2eBNi6e

16. Universidad Nacional Autónoma de México. Examen Médico Automatizado. México: UNAM; 2015.

17. Escuela Nacional de Enfermería y Obstetricia. Examen Médico Automatizado. México: ENEOUNAM; 2014.

18. Hernández-Zamora ZE, Cruz-Juárez A. Conductas sexuales riesgosas y adictivas en estudiantes universitarios. Psicología y Salud. 2008; 18(2): 227-36. https://bit.ly/35HDh3s

19. Aguilar-Barojas S. Fórmulas para el cálculo de la muestra en investigaciones de salud. Salud Tab. 2005;11(1-2):333-8. https://ng.cl/rspoo

20. Pulido-Rull MA, Carazo-Cardona V, Orta-González Sicilia G, Coronel-Villalobos M, Vera-García F. Conducta sexual de riesgo en los estudiantes de licenciatura de la Universidad Intercontinental. Rev. intercont. psicol. educ. 2011; 13(1): 11-27. https://bit.ly/2HFoMCc 
21. Secretaría de Salud. Ley General de Salud. Título quinto. Investigación para la salud. México: SSA; Última reforma publicada DOF 12-07-2018.

22. Comisión interinstitucional de enfermería. Código de Ética para las enfermeras y enfermeros de México. México: SSA; 2001.

23. Bermúdez-González A, Cárdenas-Jiménez M, Fernández-García V, Matus-Miranda R, Pérez-Cabrera I, Olvera-Arreola SS. Principios éticos para la Investigación en la ENEO. México: UNAM-ENEO; 2013.

24. Comisión Nacional de los Derechos Humanos. Ley general de los derechos de niñas, niños y adolescentes. Ley general de prestación de servicios para la atención, cuidado y desarrollo integral infantil. México: CNDH; 2018. https://bit.ly/2p5otdZ

25. Tapia-Martínez H, González-Hernando C, Puebla-Nicolás E. Influencia de determinantes de género en la anticoncepción de estudiantes de Enfermería y Obstetricia mexicanas. Enferm. univ. 2017; 14(4): 251-8. http://dx.doi.org/10.1016/j.reu.2017.08.002

26. Somba MJ, Mbonile M, Obure J, Mahande MJ. Sexual behaviour, contraceptive knowledge and use among female undergraduates' students of Muhimbili and Dar es Salaam Universities, Tanzania: A cross-sectional study. BMC Womens Health. 2014; 14(94):1-8. https://doi.org/10.1186/1472-6874-14-94

27. Sweya MN, Msuya SE, Mahande MJ, Manongi R. Contraceptive knowledge, sexual behavior, and factors associated with contraceptive use among female undergraduate university students in Kilimanjaro region in Tanzania. Adolesc Health Med Ther. 2016; (7): 109-15.

https://doi.org/10.2147/AHMT.S108531

28. Ahmed ZD, Sule IB, Abolaji ML, Mohammed Y, Nguku P. Knowledge and utilization of contraceptive devices among unmarried undergraduate students of a tertiary institution in Kano State, Nigeria 2016. Pan Afr Med J. 2017; 26(103): 1-8. https://doi.org/10.11604/pamj.2017.26.103.11436

29. Folch C, Álvarez JL, Casabona J, Brotons M, CastellsaguéX. Determinantes de las conductas sexuales de riesgo en jóvenes de Cataluña. Rev. Esp. Salud Publica. 2015; 89(5): 471-85. http://dx.doi.org/10.4321/S1135-57272015000500005

30. Shayo FK, Kalomo MH. Prevalence and correlates of sexual intercourse among sexually active inschool adolescents: an analysis of five sub-Sahara African countries for the adolescent's sexual health policy implications. BMC Public Health. 2019; 19(1): 1-8. https://doi.org/10.1186/s12889-019-7632-1

31. Sanchez ZM, Nappo SA, Cruz JI, Carlini EA, Carlini CM, Martins SS. Sexual behavior among high school students in Brazil: Alcohol consumption and legal and illegal drug use associated with unprotected sex. Clinics. 2013; 68(4): 489-94. https://ng.cl/39jx

32. Saeteros-Hernández RC, Pérez-Piñero J, Sanabria-Ramos G. Conducta de riesgo y problemas sexuales y reproductivos de estudiantes universitarios ecuatorianos. Humanid. méd. 2015; 15(3): 421-39. https://bit.ly/2VYYoNe 